\title{
Variational results for the Rabi Hamiltonian
}

\author{
R.F. Bishop ${ }^{a}$, N.J. Davidson ${ }^{b}$, R.M. Quick ${ }^{b}$, D.M. van der Walt ${ }^{c}$ \\ a Department of Physics, University of Manchester Institute of Science and Technology, Manchester M60 IQD. UK \\ b Department of Physics, University of Pretoria, Pretoria 0002, South Africa \\ 'Department of Physics, VISTA University (Mamelodi Campus), Private Bag X 1311, Silverton 0127, South Africa
}

Received 7 November 1998; revised manuscript received 14 January 1999; accepted for publication 19 January 1999 Communicated by L.J. Sham

\begin{abstract}
We present simple two- and three-parameter variational calculations for the Rabi Hamiltonian. The importance of symmetry in the ansätze is stressed. The numerical results indicate that our ansätze provide accurate approximations both to the groundstate energy and wavefunction and to the first excited state if the two-boson energy significantly exceeds the level splitting. (C) 1999 Elsevier Science B.V.
\end{abstract}

PACS: $63.20 . \mathrm{Kr} ; 71.38 .+\mathrm{i} ; 32.90 .+\mathrm{a}$

Keywords: Two-level systems: Rabi Hamiltonian; Phonon-electron interaction; Photon-electron interaction

\section{Introduction}

Simple models of two-level systems interacting with various numbers of boson modes are of considerable interest in many fields of physics. If only one boson mode is considered, models such as the Rabi Hamiltonian and the Jaynes-Cummings model [1] (which is just the Rabi model after the rotating wave approximation (RWA) has been made) are of relevance, particularly in the areas of quantum optics [2], where the boson is a photon, and in condensed matter, where it is a phonon. These models have also been of considerable interest in the field of quantum chaos [3].

Although the Rabi model appears to be very straightforward, this impression is deceptive, and there is a wealth of physical insight to be gained from an in-depth study of Hamiltonians in this class. In particular, there is no proof that this Hamiltonian is integrable at levels beyond the RWA, although there are some isolated exact points (originally found by Judd in the context of the Jahn-Teller model [4], and which we will refer to subsequently as Juddian points) and a conjecture by Reik et al. [5] which has strong numerical support, but no rigorous proof.

There is thus considerable interest in approximate methods to determine the spectra and wavefunctions for such models. Various methods have been attempted (see for example Refs. [6,7] and references therein), with varying degrees of success. In this paper, we wish to consider a simple variational ansatz for the wavefunction for the Rabi Hamiltonian. Although some earlier variational calculations have been performed [6], errors of up to $30 \%$ are reported. Our ansätze, which take the conserved parity of the Hamiltonian into ac count, lead to very much better results, with errors in the ground-state energy (relative to results obtained from a large-scale diagonalization) of less than $1 \%$ for all values of coupling between the two-level sys- 
tem and the boson field. In addition, results for quantities other than the energy suggest that the wavefunctions themselves are good approximations to the exact wavefunction.

The remainder of the paper is organized as follows: In Section 2, we discuss the Rabi Hamiltonian in general. Section 3 contains our variational ansätze for this model, and the results obtained are presented. Finally, we draw conclusions in Section 4.

\section{The Rabi Hamiltonian}

The Rabi Hamiltonian models the interaction of a single bosonic mode with a two-level system in the dipole approximation, and is given by

$H_{\mathrm{R}}=\frac{1}{2} \omega_{0} \sigma^{z}+\omega b^{\dagger} b+g\left(\sigma^{+}+\sigma^{-}\right)\left(b^{\dagger}+b\right)$,

where $\sigma^{ \pm} \equiv \sigma^{x} \pm i \sigma^{y}$ and $\sigma^{x}, \sigma^{y}$ and $\sigma^{z}$ are Pauli matrices, and $b$ and $b^{\dagger}$ are boson annihilation and creation operators respectively, obeying the commutation relation $\left[b, b^{\dagger}\right]=1$. The frequency of the boson mode is indicated by $\omega$, the energy splitting in the two-level system by $\omega_{0}$, and the coupling between the system and the field by $g$.

There is a conserved parity $\Pi$ associated with the Hamiltonian (1),

$\Pi \equiv \exp [\mathrm{i} \pi(N)], \quad N \equiv b^{\dagger} b+\frac{1}{2}\left(\sigma^{z}+1\right)$.

It is easy to see that $N$ essentially counts the number of "excitations" in the complete system. Note that if the RWA is made, i.e. if one omits the counter-rotating terms $g\left(b^{\dagger} \sigma^{+}+b \sigma^{-}\right)$, then $\left[H_{\mathrm{RWA}}, N\right]=0$, thereby permitting an exact solution in terms of a series of diagonalizations of $2 \times 2$ matrices.

The Rabi Hamiltonian (1) has some exact limits. For zero coupling $(g=0)$ and $\omega_{0}>0$, the exact ground state is given by $|0\rangle|\downarrow\rangle$, where the first ket refers to the boson mode in occupation number representation and the second to the lower eigenstate of the two-level system, with $E_{g}(g=0)=-\frac{1}{2} \omega_{0}$. For the case of two degenerate levels $\left(\omega_{0}=0\right)$, the positiveand negative-parity ground states are degenerate, implying that the ground state does not have good parity in this limit for any coupling. In this limit the exact ground states are coherent boson states multiplied by eigenfunctions of $\sigma^{x}$,

$$
\begin{aligned}
& \left|\Psi_{ \pm}\right\rangle=\frac{1}{\sqrt{2}} \exp \left[-\frac{2 g^{2}}{\omega^{2}}\right] \exp \left[ \pm \frac{2 g}{\omega} b^{\dagger}\right]|0\rangle(|\downarrow\rangle \mp|\uparrow\rangle) \\
& =\frac{1}{\sqrt{2}} \exp \left[-\frac{2 g^{2}}{\omega^{2}}\right] \exp \left[ \pm \frac{2 g}{\omega} b^{\dagger}\right]|0\rangle \exp \left[\mp \frac{\sigma^{+}}{2}\right]|\downarrow\rangle
\end{aligned}
$$

The ground-state energy in this case is given by $E_{g}\left(\omega_{0}=0\right)=-4 g^{2} / \omega$. The case $\omega_{0}=0$ is important because the Rabi Hamiltonian with a finite value for $\omega_{0}$ goes over to this case as $g \rightarrow \infty$.

\section{Variational Ansätze for the Rabi Hamiltonian}

From the previous section, it is clear that the groundstate wavefunction of the Rabi Hamiltonian in the limit $\omega_{0}=0$ is a product of a coherent state characterized by the parameter $2 g / \omega$ and a rotation of the down eigenstate of $\sigma^{2}$. As an initial variational ansatz, one can consider the normalized state given by

$\left|\Psi_{\mathrm{V}}(x, y)\right\rangle=\frac{1}{\sqrt{y^{2}+1}}|x\rangle \exp \left[\frac{1}{2} y \sigma^{+}\right]|\downarrow\rangle$,

where the normalized coherent state $|x\rangle$ is given by

$|x\rangle=\exp \left[x b^{\dagger}-\frac{1}{2} x^{2}\right]|0\rangle$.

Clearly, Eq. (3) corresponds to the cases $x= \pm 2 \mathrm{~g} / \omega$ and $y=\mp 1$. One can now determine $x$ and $y$ by minimizing the expectation value of the Hamiltonian (1) in this state. Since this procedure is however entirely equivalent to the variational method previously given by Qin et al. [6] one obtains identical results to those reported in their paper.

To obtain an estimate of the accuracy of the variational results, we have determined the spectrum of the Rabi Hamiltonian by means of a large-scale diagonalization of $H_{\mathrm{R}}$ in a space of 201 states which are of the form of products of oscillator states and eigenstates of $\sigma^{z}$. We have checked that the diagonalization has converged at each value of $g$ of interest, and have also checked the energies obtained from the diagonalization against the exact Juddian points. Excellent agreement is obtained. (Note that there are no Juddian points for the ground state of the Rabi Hamiltonian; our checks have therefore been made against excited states. The ground-state results should be at least as accurate, although no formal proof for this conjecture exists.) 
One can then compare the variational results using the ansatz (4) with those from the diagonalization, and it is evident from the paper of Qin ct al. [6] that the agreement is qualitatively good, but not remarkable from a quantitative viewpoint. To improve the results, we need to re-examine the ansatz (4). The important omission in this ansatz is the question of parity. The ansatz (4) is of mixed parity, while the ground state of the Rabi Hamiltonian with a non-zero level splitting (i.c. $\omega_{0} \neq 0$ ) is of positive parity. (This is simplest to see at $g=0$, where the exact ground state is simply $|0\rangle|\downarrow\rangle$.) To improve our variational ansatz, we therefore construct a state of positive parity from Eq. (4) by projecting as follows,

$$
\begin{gathered}
\left|\Psi_{\mathrm{V}}^{(\mathrm{p})}(x, y)\right\rangle=(1+\Pi)\left|\Psi_{\mathrm{V}}(x, y)\right\rangle \\
\quad=\left|\Psi_{\mathrm{V}}(x, y)\right\rangle+\left|\Psi_{\mathrm{V}}(-x,-y)\right\rangle,
\end{gathered}
$$

which obviously has positive parity since $\Pi^{2}=1$. Note that the simultaneous replacement of $x$ by $-x$ and $y$ by $-y$ leaves the state $\left|\Psi_{\mathrm{V}}^{(\mathrm{p})}(x, y)\right\rangle$ unchanged. At the same time, we obtain an ansatz for the lowestlying negative-parity state (the first excited state of the Rabi Hamiltonian) from the parity partner of (6),

$\left|\Psi_{\mathrm{V}}^{(\mathrm{n})}(x, y)\right\rangle=(1-\Pi)\left|\Psi_{\mathrm{V}}(x, y)\right\rangle$.

These states can be written jointly in explicit form as

$$
\begin{aligned}
& \left|\Psi_{\mathrm{V}}^{(\mathrm{p} . n)}(x, y)\right\rangle=[\{|x\rangle \pm|-x\rangle\}|\downarrow\rangle \\
& \quad+y\{|x\rangle \mp|-x\rangle\}|\uparrow\rangle],
\end{aligned}
$$

where the upper (lower) sign refers to the positive (negative) parity ansatz.

For numerical reasons, it turns out to be more convenient to normalize the two terms in braces in (8) before taking the linear combination, to obtain the normalized state

$$
\begin{aligned}
&\left|\Psi_{\mathrm{V}}^{(\mathrm{p}, \mathrm{n})}(x, v)\right\rangle=A_{\iota}\left[A_{ \pm}\{|x\rangle \pm|-x\rangle\}|\downarrow\rangle\right. \\
&\left.+v A_{\mp}\{|x\rangle \mp|-x\rangle\}|\uparrow\rangle\right]
\end{aligned}
$$

where

$A_{i}=\left[l^{2}+1\right]^{-1 / 2}$, $A_{ \pm}=\left[2\left(1 \pm \exp \left[-2 x^{2}\right]\right)\right]^{-1 / 2}$.

Comparison of (8) and (9) shows that they are physically entirely equivalent.
The expectation values of the Hamiltonian (1) in these two states,

$$
\begin{aligned}
& \left\langle\Psi_{\mathrm{V}}^{(\mathrm{p}, \mathrm{n})}(x, v)\left|H_{\mathrm{R}}\right| \Psi_{\mathrm{V}}^{(\mathrm{p}, \mathrm{n})}(x, v)\right\rangle=\frac{\omega_{0}}{2} \frac{v^{2}-1}{v^{2}+1} \\
& +\omega \frac{x^{2}}{v^{2}+1}\left(\tanh \left[x^{2}\right]^{ \pm 1}+v^{2} \operatorname{coth}\left[\left.x^{2}\right|^{ \pm 1}\right)\right. \\
& +\frac{1}{v^{2}+1} \frac{8 g x v}{\sqrt{1-\exp \left[-4 x^{2}\right]}},
\end{aligned}
$$

can now be made stationary with respect to $x$ and $v$ and the cnergy cvaluated. This corresponds to a projection before variation (PBV). The minimization with respect to $v$ yields a quadratic equation for $v_{\text {opt }}$ in terms of $x_{\mathrm{opt}}$, leaving a single non-linear equation for $x_{\mathrm{opt}}$ to be solved numerically.

The results of the calculation of the ground-state energy are shown in Table 1. As is clear from our results, the quantitative agreement between the diagonalization and the PBV calculation based on the ansatz (9) is excellent. Considering the computational ease of the minimization, the ansatz (9) constitutes a very simple and straightforward way to generate the ground-state energy of the Rabi Hamiltonian to high accuracy. It is also clear from Table 1 that the quantities $x_{\mathrm{opt}}$ and $y_{\mathrm{opt}}$ go smoothly from their $g=0$ values of 0 to their large$g$ values of $2 g / \omega$ and $-\frac{1}{2}$ respectively (corresponding to the solution ( 3$)$ ) as $g$ becomes large.

Table 2 contains our results for a PBV calculation using the negative-parity ansatz for the first excited state. The results are good, although not in general at the same level of accuracy as those for the ground state. Possible reasons for this discrepancy will be discussed in the next section.

It is possible to obtain a three-parameter ansatz for the Rabi ground state and first excited state wavefunctions from the two-parameter ansätze ( 9 ) by noting that there is no a priori reason for assuming that the two combinations of coherent boson states need have the same parameter $x$, and we therefore propose the following as a three-parameter ansatz for the Rabi Hamiltonian,

$$
\begin{aligned}
& \left|\Psi_{\mathrm{V}}^{(\rho, \pi)}\left(x_{1}, x_{2}, v\right)\right\rangle=A_{l}\left[A_{1 . \pm}\left\{\left|x_{1}\right\rangle \pm\left|-x_{1}\right\rangle\right\}|\downarrow\rangle\right. \\
& \left.\quad+v A_{2, \mp}\left\{\left|x_{2}\right\rangle \mp\left|-x_{2}\right\rangle\right\}|\uparrow\rangle\right]
\end{aligned}
$$

where 
Table 1

Comparison of the ground-state energy of the Rabi Hamiltonian obtained from the two-parameter PBV ansatz (9), $E_{\mathrm{V} .0}^{\mathrm{P2}}$, with the results of a large-scale diagonalization, $E_{\mathrm{D}, 0}$. Also shown are the percentage error $\left|E_{\mathrm{V}, 0}^{\mathrm{P} 2}-E_{\mathrm{D}, 0}\right| / E_{\mathrm{D}, 0} \times 100$ and the values of the variational parameters $x_{\mathrm{opt}}$ and $y_{\mathrm{opt}}$ at the stationary point of the energy.

\begin{tabular}{|c|c|c|c|c|c|c|c|}
\hline$\omega_{1}$ & $\omega$ & $g$ & $E_{\mathrm{V}, 0}^{\mathrm{P} 2}$ & $E_{\mathrm{D}, 0}$ & $\%$ Error & $x_{\mathrm{opl}}$ & lopt \\
\hline 1.0 & 1.0 & $\begin{array}{l}0.05 \\
0.1 \\
0.2 \\
0.5 \\
1.0 \\
2.0 \\
5.0\end{array}$ & $\begin{array}{l}-5.05012 \times 10^{-1} \\
-5.20201 \times 10^{-1} \\
-5.83285 \times 10^{-1} \\
-1.14211 \\
-4.01580 \\
-1.60039 \times 10^{1} \\
-1.00001 \times 10^{2}\end{array}$ & $\begin{array}{l}-5.05013 \times 10^{-1} \\
-5.20202 \times 10^{-1} \\
-5.83327 \times 10^{-1} \\
-1.14795 \\
-4.01693 \\
-1.60040 \times 10^{1} \\
-1.00001 \times 10^{2}\end{array}$ & $\begin{array}{r}2 \times 10^{-4} \\
2 \times 10^{-4} \\
7 \times 10^{-3} \\
5 \times 10^{-1} \\
3 \times 10^{-2} \\
6 \times 10^{-4} \\
<1 \times 10^{-4}\end{array}$ & $\begin{array}{r}0.071 \\
0.142 \\
0.291 \\
0.859 \\
1.995 \\
4.000 \\
10.000\end{array}$ & $\begin{array}{l}-0.050 \\
-0.101 \\
-0.208 \\
-0.626 \\
-0.939 \\
-0.984 \\
-0.998\end{array}$ \\
\hline 1.0 & 2.0 & $\begin{array}{l}0.05 \\
0.1 \\
0.2 \\
0.5 \\
1.0 \\
2.0 \\
5.0\end{array}$ & $\begin{array}{l}-5.03334 \times 10^{-1} \\
-5.13362 \times 10^{-1} \\
-5.53807 \times 10^{-1} \\
-8.51754 \times 10^{-1} \\
-2.10416 \\
-8.00798 \\
-5.00012 \times 10^{1}\end{array}$ & $\begin{array}{l}-5.03335 \times 10^{-1} \\
-5.13363 \times 10^{-1} \\
-5.53809 \times 10^{-1} \\
-8.51992 \times 10^{-1} \\
-2.10825 \\
-8.00855 \\
-5.00013 \times 10^{1}\end{array}$ & $\begin{array}{l}2 \times 10^{-4} \\
2 \times 10^{-4} \\
4 \times 10^{-4} \\
3 \times 10^{-2} \\
2 \times 10^{-1} \\
7 \times 10^{-3} \\
2 \times 10^{-4}\end{array}$ & $\begin{array}{l}0.041 \\
0.082 \\
0.164 \\
0.424 \\
0.938 \\
1.999 \\
5.000\end{array}$ & $\begin{array}{l}-0.033 \\
-0.067 \\
-0.135 \\
-0.416 \\
-0.747 \\
-0.969 \\
-0.995\end{array}$ \\
\hline 2.0 & 1.0 & $\begin{array}{l}0.05 \\
0.1 \\
0.2 \\
0.5 \\
1.0 \\
2.0 \\
5.0\end{array}$ & $\begin{array}{l}-1.00334 \\
-1.01345 \\
-1.05530 \\
-1.42799 \\
-4.06288 \\
-1.60156 \times 10^{1} \\
-1.00002 \times 10^{2}\end{array}$ & $\begin{array}{l}-1.00334 \\
-1.01345 \\
-1.05533 \\
-1.43655 \\
-4.06746 \\
-1.60159 \times 10^{1} \\
-1.00003 \times 10^{2}\end{array}$ & $\begin{array}{r}<1 \times 10^{-4} \\
<1 \times 10^{-4} \\
3 \times 10^{-3} \\
6 \times 10^{-1} \\
1 \times 10^{-1} \\
2 \times 10^{-3} \\
1 \times 10^{-3}\end{array}$ & $\begin{array}{r}0.058 \\
0.116 \\
0.237 \\
0.699 \\
1.983 \\
3.998 \\
10.000\end{array}$ & $\begin{array}{l}-0.033 \\
-0.067 \\
-0.138 \\
-0.430 \\
-0.882 \\
-0.969 \\
-0.995\end{array}$ \\
\hline
\end{tabular}

$A_{i}=\left[v^{2}+1\right]^{-1 / 2}$,

$A_{1, \pm}=\left[2\left(1 \pm \exp \left[-2 x_{1}^{2}\right]\right)\right]^{-1 / 2}$,

$A_{2, \pm}=\left[2\left(1 \pm \exp \left[-2 x_{2}^{2}\right]\right)\right]^{-1 / 2}$,

and where the upper (lower) sign refers to the positive (negative) parity ansatz. We note here that the ansatz (11) is clearly invariant under the replacement $x_{1} \rightarrow$ $-x_{1}$ as well as under the simultaneous replacements $x_{2} \rightarrow-x_{2}, v \rightarrow-v$. For convenience, we consider here only the case $x_{1}, x_{2} \geqslant 0$.

The expectation values of the Hamiltonian (1) in these states are given by

$$
\begin{aligned}
& \left\langle\Psi_{\mathrm{V}}^{(\mathrm{p}, \mathrm{n})}\left(x_{1}, x_{2}, v\right)\left|H_{\mathrm{R}}\right| \Psi_{\mathrm{V}}^{(\mathrm{p}, \mathrm{n})}\left(x_{1}, x_{2}, v\right)\right\rangle \\
& \quad=A_{i}^{2}\left[\frac{1}{2} \omega_{0}\left(v^{2}-1\right)+\omega\left(x_{1}^{2} \tanh \left[x_{1}^{2}\right] \pm 1\right.\right. \\
& \left.\left.\quad+v^{2} x_{2}^{2} \operatorname{coth}\left[x_{2}^{2}\right]^{ \pm 1}\right)+8 g v B_{ \pm}\left(x_{1}, x_{2}\right)\right], \\
& B_{ \pm}\left(x_{1}, x_{2}\right) \\
& \quad=A_{1, \pm} A_{2, \mp}\left\{\left(x_{2}+x_{1}\right) \exp \left[-\frac{1}{2}\left(x_{1}-x_{2}\right)^{2}\right]\right. \\
& \left.\quad \pm\left(x_{2}-x_{1}\right) \exp \left[-\frac{1}{2}\left(x_{1}+x_{2}\right)^{2}\right]\right\},
\end{aligned}
$$

where $A_{i}, A_{1, \pm}$ and $A_{2 . \pm}$ are as given below (11). The minimization with respect to $v$ yields a quadratic equation for $v_{\mathrm{opt}}$ in terms of $x_{1, \mathrm{opt}}$ and $x_{2, \mathrm{opt}}$. The solution with the lowest energy is always given by

$v_{\mathrm{opt}}=\frac{\omega_{0}+\omega C_{ \pm}}{8 g B_{ \pm}}-\sqrt{1+\left(\frac{\omega_{0}+\omega C_{ \pm}}{8 g B_{ \pm}}\right)^{2}}$,

where

$C_{ \pm}\left(x_{1}, x_{2}\right)=-x_{1}^{2} \tanh \left[x_{1}^{2}\right]^{ \pm 1} \mid x_{2}^{2} \operatorname{coth}\left[x_{2}^{2}\right]^{ \pm 1}$

We are thus left with two coupled non-linear equations for $x_{1, \mathrm{opt}}$ and $x_{2, \mathrm{opt}}$ to be solved numerically. We note that there are classes of solutions with $x_{1, \mathrm{opt}}=0$ (ground state) and $x_{2, \mathrm{opt}}=0$ (excited state), but these do not minimize the energies, and so are not considered.

In the limit of very small couplings, it is possible to obtain asymptotic expressions for the parameters $v^{\prime}$ opt, $x_{1, \mathrm{opt}}$ and $x_{2, \mathrm{opt}}$, for the ground state, 
Table 2

Comparison of the energy of the first excited state of the Rabi Hamiltonian obtained from the two-parameter PBV ansatz (9), $E_{\mathrm{V} .1}^{\mathrm{P2}}$, with the results of a large-scale diagonalization, $E_{\mathrm{D}, 1}$. Also shown are the percentage error $\left|E_{\mathrm{V}, 1}^{\mathrm{P} 2}-E_{\mathrm{D}, 1}\right| / E_{\mathrm{D}, 1} \times 100$ and the values of the variational parameters $x_{\mathrm{opt}}$ and $y_{\mathrm{opt}}$ at the stationary point of the energy.

\begin{tabular}{|c|c|c|c|c|c|c|c|}
\hline$\omega_{0}$ & $\omega$ & $g$ & $E_{\mathrm{V}, 1}^{\mathrm{P} 2}$ & $E_{1.1}$ & $\%$ Error & $x_{1, p 1}$ & $l_{\text {ipp }}$ \\
\hline \multirow[t]{7}{*}{1.0} & 1.0 & 0.05 & $3.96137 \times 10^{-1}$ & $3.95102 \times 10^{-1}$ & $3 \times 10^{-1}$ & 0.279 & -0.982 \\
\hline & & 0.1 & $2.84083 \times 10^{-1}$ & $2.80666 \times 10^{-1}$ & 1.2 & 0.401 & -0.964 \\
\hline & & 0.2 & $3.24806 \times 10^{-2}$ & $2.33675 \times 10^{-2}$ & 40 & 0.590 & -0.932 \\
\hline & & 0.5 & $-9.98782 \times 10^{-1}$ & -1.01018 & 1.1 & 1.067 & -0.885 \\
\hline & & 1.0 & -4.01545 & -4.01658 & $3 \times 10^{-2}$ & 1.997 & -0.940 \\
\hline & & 2.0 & $-1.60039 \times 10^{1}$ & $-1.60040 \times 10^{1}$ & $6 \times 10^{-4}$ & 4.000 & -0.984 \\
\hline & & 5.0 & $-1.00001 \times 10^{2}$ & $-1.00001 \times 10^{2}$ & $<1 \times 10^{-4}$ & 10.000 & -0.998 \\
\hline \multirow[t]{7}{*}{1.0} & 2.0 & 0.05 & $4.90049 \times 10^{-1}$ & $4.90049 \times 10^{-1}$ & $<1 \times 10^{-4}$ & 0.070 & -10.050 \\
\hline & & 0.1 & $4.60760 \times 10^{-1}$ & $4.60758 \times 10^{-1}$ & $4 \times 10^{-4}$ & 0.140 & -5.098 \\
\hline & & 0.2 & $3.50617 \times 10^{-1}$ & $3.50542 \times 10^{-1}$ & $2 \times 10^{-2}$ & 0.270 & -2.864 \\
\hline & & 0.5 & $-2.69004 \times 10^{-1}$ & $-2.71650 \times 10^{-1}$ & 1 & 0.595 & -1.355 \\
\hline & & 1.0 & -1.96664 & -1.97218 & $3 \times 10^{-1}$ & 1.042 & -1.000 \\
\hline & & 2.0 & -8.00764 & -8.00821 & $7 \times 10^{-3}$ & 1.999 & -0.970 \\
\hline & & 5.0 & $-5.00013 \times 10^{1}$ & $-5.00013 \times 10^{1}$ & $<1 \times 10^{-4}$ & 5.000 & -0.995 \\
\hline \multirow[t]{7}{*}{2.0} & 1.0 & 0.05 & $-1.01956 \times 10^{-2}$ & $-1.65009 \times 10^{-2}$ & 38 & 0.173 & -0.102 \\
\hline & & 0.1 & $-4.28514 \times 10^{-2}$ & $-6.42075 \times 10^{-2}$ & 33 & 0.340 & -0.212 \\
\hline & & 0.2 & $-1.87367 \times 10^{-1}$ & $-2.35841 \times 10^{-1}$ & 21 & 0.601 & -0.419 \\
\hline & & 0.5 & -1.11473 & -1.15708 & 3.8 & 1.079 & -0.706 \\
\hline & & 1.0 & -4.06213 & -4.06664 & $1 \times 10^{-1}$ & 1.986 & -0.882 \\
\hline & & 2.0 & $-1.60156 \times 10^{1}$ & $-1.60159 \times 10^{1}$ & $2 \times 10^{-4}$ & 3.998 & -0.969 \\
\hline & & 5.0 & $-1.00002 \times 10^{2}$ & $-1.00003 \times 10^{2}$ & $1 \times 10^{-4}$ & 10.000 & -0.995 \\
\hline
\end{tabular}

$$
\begin{aligned}
& v_{\mathrm{opt}} \rightarrow \frac{-2 g}{\omega+\omega_{0}}, \quad x_{1, \mathrm{opt}} \rightarrow \frac{2 g}{\omega}\left\{1+\frac{\omega_{0}}{\omega}\right\}^{-1 / 2}, \\
& x_{2, \mathrm{opt}} \rightarrow \frac{2 g}{\omega}\left\{1+\frac{\omega_{0}}{3 \omega}\right\}^{-1 / 2},
\end{aligned}
$$

which yield good starting values for the numerical solution routines.

The situation for the first excited state is more complex, and depends explicitly on whether the system is sub- or supra-resonant. In fact, for the sub-resonant case $\left(\omega<\omega_{0}\right)$, the asymptotic forms are the solutions of transcendental equations; however, one can show that in the limit of zero coupling, $x_{1, \text { opt }}$ and $v_{\text {opt }}$ are zero, but $x_{2, \text { opt }}$ is a non-zero constant dependent on the frequencies. For the other two cases, the asymptotic expression are

$$
\begin{aligned}
& v_{\mathrm{opt}} \rightarrow-1, \quad x_{1, \mathrm{opt}} \rightarrow \frac{\sqrt{6} g}{\omega}, \\
& x_{2, \mathrm{opt}} \rightarrow \sqrt{\frac{2 g}{\omega}},
\end{aligned}
$$

in the case $\omega_{0}=\omega$, and

$$
\begin{aligned}
& v_{\mathrm{opt}} \rightarrow \frac{\omega_{0}-\omega}{2 g}, \quad x_{1, \mathrm{opt}} \rightarrow \frac{2 g}{\omega}\left\{1-\frac{\omega_{0}}{3 \omega}\right\}^{-1 / 2}, \\
& x_{2, \mathrm{opt}} \rightarrow \frac{2 g}{\omega}\left\{1-\frac{\omega_{0}}{\omega}\right\}^{-1 / 2},
\end{aligned}
$$

in the case $\omega_{0}<\omega$.

Our results for the energies for these threeparameter ansätze are shown in Tables 3 and 4 and Figs. 1 and 2. It is clear from the values presented that we have obtained a very accurate approximation for both the ground-state and first excited-state energies of the Rabi Hamiltonian with very little numerical effort.

As with any variational calculation, there is the question of the quality of the wavefunction itself. Although variational calculations are useful in determining energies, particularly due to the upper-bound nature of their results, there is no guarantee that the wavefunction itself is accurate. A failure of the wavefunction is usually revealed in the calculation of expecta- 
Table 3

Comparison of the ground-state energy of the Rabi Hamiltonian obtained from the three-parameter PBV ansatz (11), $E_{\mathrm{V}, 0}^{\mathrm{P} 3}$, with the results of a large-scale diagonalization, $E_{\mathrm{D}, 0}$. Also shown are the percentage error $\left|E_{\mathrm{V}, 0}^{\mathrm{P} 3}-E_{\mathrm{D}, 0}\right| / E_{\mathrm{D}, 0} \times 100$ and the values of the variational parameters $x_{1, \mathrm{opt}}, x_{2, \mathrm{opt}}$ and $y_{\mathrm{opl}}$ at the stationary point of the energy.

\begin{tabular}{|c|c|c|c|c|c|c|c|c|}
\hline$\omega_{0}$ & $\omega$ & $g$ & $E_{\mathrm{V}, 0}^{\mathrm{P3}}$ & $E_{\mathrm{D}, 0}$ & $\%$ Error & $x_{1, \mathrm{opt}}$ & $x_{2, \mathrm{opt}}$ & rupt \\
\hline 1.0 & 1.0 & $\begin{array}{l}0.05 \\
0.1 \\
0.2 \\
0.5 \\
1.0 \\
2.0 \\
5.0\end{array}$ & $\begin{array}{l}-5.05013 \times 10^{-1} \\
-5.20202 \times 10^{-1} \\
-5.83326 \times 10^{-1} \\
-1.14676 \\
-4.01677 \\
-1.60040 \times 10^{1} \\
-1.00001 \times 10^{2}\end{array}$ & $\begin{array}{l}-5.05013 \times 10^{-1} \\
-5.20202 \times 10^{-1} \\
-5.83327 \times 10^{-1} \\
-1.14795 \\
-4.01693 \\
-1.60040 \times 10^{1} \\
-1.00001 \times 10^{2}\end{array}$ & $\begin{array}{r}<1 \times 10^{-4} \\
<1 \times 10^{-4} \\
2 \times 10^{-4} \\
1 \times 10^{-1} \\
\quad 4 \times 10^{-3} \\
<1 \times 10^{-4} \\
<1 \times 10^{-4}\end{array}$ & $\begin{array}{r}0.071 \\
0.142 \\
0.292 \\
0.856 \\
1.987 \\
3.999 \\
10.000\end{array}$ & $\begin{array}{r}0.087 \\
0.174 \\
0.351 \\
0.942 \\
2.003 \\
4.000 \\
10.000\end{array}$ & $\begin{array}{l}-0.050 \\
-0.101 \\
-0.208 \\
-0.618 \\
-0.935 \\
-0.984 \\
-0.997\end{array}$ \\
\hline 1.0 & 2.0 & $\begin{array}{l}0.05 \\
0.1 \\
0.2 \\
0.5 \\
1.0 \\
2.0 \\
5.0\end{array}$ & $\begin{array}{l}-5.03334 \times 10^{-1} \\
-5.13363 \times 10^{-1} \\
-5.53809 \times 10^{-1} \\
-8.51976 \times 10^{-1} \\
-2.10739 \\
-8.00847 \\
-5.00013 \times 10^{1}\end{array}$ & $\begin{array}{l}-5.03335 \times 10^{-1} \\
-5.13363 \times 10^{-1} \\
-5.53809 \times 10^{-1} \\
-8.51992 \times 10^{-1} \\
-2.10825 \\
-8.00855 \\
-5.00013 \times 10^{1}\end{array}$ & $\begin{array}{r}2 \times 10^{-4} \\
<1 \times 10^{-4} \\
<1 \times 10^{-4} \\
2 \times 10^{-3} \\
4 \times 10^{-2} \\
1 \times 10^{-3} \\
<1 \times 10^{-4}\end{array}$ & $\begin{array}{l}0.041 \\
0.082 \\
0.164 \\
0.424 \\
0.932 \\
1.995 \\
5.000\end{array}$ & $\begin{array}{l}0.046 \\
0.093 \\
0.186 \\
0.470 \\
0.977 \\
2.003 \\
5.000\end{array}$ & $\begin{array}{l}-0.033 \\
-0.067 \\
-0.135 \\
-0.351 \\
-0.738 \\
-0.967 \\
-0.995\end{array}$ \\
\hline 2.0 & 1.0 & $\begin{array}{l}0.05 \\
0.1 \\
0.2 \\
0.5 \\
1.0 \\
2.0 \\
5.0\end{array}$ & $\begin{array}{l}-1.00334 \\
-1.01345 \\
-1.05533 \\
-1.43491 \\
-4.06678 \\
-1.60159 \times 10^{1} \\
-1.00003 \times 10^{2}\end{array}$ & $\begin{array}{l}-1.00334 \\
-1.01345 \\
-1.05533 \\
-1.43655 \\
-4.06746 \\
-1.60159 \times 10^{1} \\
-1.00003 \times 10^{2}\end{array}$ & $\begin{aligned}< & 1 \times 10^{-4} \\
< & 1 \times 10^{-4} \\
< & 1 \times 10^{-4} \\
& 1 \times 10^{-1} \\
& 2 \times 10^{-2} \\
< & 1 \times 10^{-4} \\
< & 1 \times 10^{-4}\end{aligned}$ & $\begin{array}{r}0.058 \\
0.116 \\
0.238 \\
0.712 \\
1.966 \\
3.996 \\
10.000\end{array}$ & $\begin{array}{r}0.078 \\
0.155 \\
0.314 \\
0.857 \\
1.998 \\
4.000 \\
10.000\end{array}$ & $\begin{array}{l}-0.033 \\
-0.067 \\
-0.138 \\
-0.432 \\
-0.875 \\
-0.969 \\
-0.995\end{array}$ \\
\hline
\end{tabular}

tion values of quantities other than the Hamiltonian. To examine the quality of our ground-state ansätze (9) and (11), we have therefore calculated the expectation values of $\sigma^{z}$ and $b^{\dagger} b$ in these states. The former value is useful as it provides an indication of when the two-level system changes from being predominantly in one eigenstate of $\sigma^{z}$ to being in a mixture of the two eigenstates, while the latter quantifies the average boson number in the system.

In the unprojected ansatz (4), the expectation values of $\sigma^{z}$ and $b^{\dagger} b$ are given by

$\left\langle\sigma^{z}\right\rangle=\frac{y^{2}-1}{y^{2}+1}$

and

$$
\left\langle b^{\dagger} b\right\rangle=x^{2} \text {. }
$$

For the two-parameter projected ansatz for the ground state (9), the relevant expectation values are given by

$$
\left\langle\sigma^{2}\right\rangle=\left(\frac{v^{2}-1}{v^{2}+1}\right)
$$

and

$$
\left\langle b^{\dagger} b\right\rangle=\frac{1}{v^{2}+1}\left(x^{2} \tanh \left[x^{2}\right]+v^{2} x^{2} \operatorname{coth}\left[x^{2}\right]\right) \text {. }
$$

The addition of the third parameter to the variational ansatz for the ground state (11) leads to an expression identical to (19) for $\left\langle\sigma^{z}\right\rangle$ and

$\left\langle b^{\dagger} b\right\rangle=\frac{1}{v^{2}+1}\left(x_{1}^{2} \tanh \left[x_{1}^{2}\right]+v^{2} x_{2}^{2} \operatorname{coth}\left[x_{2}^{2}\right]\right)$.

Our results for $\left\langle\sigma^{z}\right\rangle$ are shown in Fig. 3 for the diagonalization results and the variational calculations, and the corresponding results for $\left\langle b^{\dagger} b\right\rangle$ in Fig. 4. In Fig. 3 , the results for the three-parameter calculation lie very close to the diagonalization, and are almost indistinguishable on a plot over this range of couplings. The dramatic improvement obtained by projecting is clearly seen, however. The agreement for $\left\langle b^{\dagger} b\right\rangle$ is even better, and the differences between the diagonalization, two-parameter and three-parameter calculations 
Table 4

Comparison of the energy of the first excited state of the Rabi Hamiltonian obtained from the three-parameter PBV ansatz (I1), $E_{\mathrm{V} .1}^{\mathrm{*}, 3}$. with the results of a large-scale diagonalization, $E_{\mathrm{D} .1}$. Also shown are the percentage error $\left|E_{\mathrm{V} .1}^{\mathrm{P} 3}-E_{\mathrm{Q}, 1.1}\right| / E_{\mathrm{l}) .1} \times 100$ and the values of the variational parameters $x_{1.0 p l}, x_{2.0 p t}$ and $y_{\mathrm{opt}}$ at the stationary point of the energy.

\begin{tabular}{|c|c|c|c|c|c|c|c|c|}
\hline$\omega_{0}$ & $\omega$ & $g$ & $E_{\mathrm{V}, 1}^{\mathrm{P3}}$ & $E_{\mathrm{D}, 1}$ & $\%$ Error & $x_{1.0 \mathrm{pl}}$ & $r_{2 . .1 p t}$ & lin \\
\hline \multirow[t]{7}{*}{1.0} & \multirow[t]{7}{*}{1.0} & 0.05 & $3.95108 \times 10^{-1}$ & $3.95102 \times 10^{-1}$ & $2 \times 10^{-3}$ & 0.119 & 0.316 & -0.956 \\
\hline & & 0.1 & $2.80737 \times 10^{-1}$ & $2.80666 \times 10^{-1}$ & $2.5 \times 10^{-2}$ & 0.230 & 0.448 & -0.922 \\
\hline & & 0.2 & $2.39619 \times 10^{-2}$ & $2.33675 \times 10^{-2}$ & 2.5 & 0.437 & 0.639 & -0.877 \\
\hline & & 0.5 & -1.00774 & -1.01018 & $2.4 \times 10^{-1}$ & 1.005 & 1.097 & -0.853 \\
\hline & & 1.0 & -4.01643 & -4.01658 & $3 \times 10^{-3}$ & 1.989 & 2.004 & -0.936 \\
\hline & & 2.0 & $-1.60040 \times 10^{1}$ & $-1.60040 \times 10^{1}$ & $<1 \times 10^{-4}$ & 3.999 & 4.000 & -0.984 \\
\hline & & 5.0 & $-1.00001 \times 10^{2}$ & $-1.00001 \times 10^{2}$ & $<1 \times 10^{-4}$ & 10.000 & 10.000 & -0.997 \\
\hline \multirow[t]{7}{*}{1.0} & \multirow[t]{7}{*}{2.0} & 0.05 & $4.90049 \times 10^{-1}$ & $4.90049 \times 10^{-1}$ & $<1 \times 10^{-4}$ & 0.055 & 0.071 & -10.050 \\
\hline & & 0.1 & $4.60758 \times 10^{-1}$ & $4.60758 \times 10^{-1}$ & $<1 \times 10^{-4}$ & 0.109 & 0.140 & -5.097 \\
\hline & & 0.2 & $3.50544 \times 10^{-1}$ & $3.50542 \times 10^{-1}$ & $5 \times 10^{-4}$ & 0.217 & 0.271 & -2.680 \\
\hline & & 0.5 & $-2.71436 \times 10^{-1}$ & $-2.71650 \times 10^{-1}$ & $8 \times 10^{-2}$ & 0.526 & 0.603 & -1.333 \\
\hline & & 1.0 & -1.97098 & -1.97218 & $6 \times 10^{-2}$ & 1.009 & 1.055 & -0.983 \\
\hline & & 2.0 & -8.00813 & -8.00821 & $1 \times 10^{-3}$ & 1.995 & 2.003 & -0.968 \\
\hline & & 5.0 & $-5.00013 \times 10^{1}$ & $-5.00013 \times 10^{1}$ & $<1 \times 10^{-1}$ & 5.000 & 5.000 & -0.995 \\
\hline \multirow[t]{7}{*}{2.0} & \multirow[t]{7}{*}{1.0} & 0.05 & $-1.61742 \times 10^{-2}$ & $-1.65009 \times 10^{-2}$ & 1.9 & 0.104 & 0.774 & -0.110 \\
\hline & & 0.1 & $-6.29720 \times 10^{-2}$ & $-6.42075 \times 10^{-2}$ & 1.9 & 0.207 & 0.791 & -0.212 \\
\hline & & 0.2 & $-2.31762 \times 10^{-1}$ & $-2.35841 \times 10^{-1}$ & 1.7 & 0.405 & 0.849 & -0.379 \\
\hline & & 0.5 & -1.14872 & -1.15708 & $7 \times 10^{-1}$ & 0.966 & 1.147 & -0.658 \\
\hline & & 1.0 & -4.06603 & -4.06664 & $2 \times 10^{-2}$ & 1.969 & 2.000 & -0.875 \\
\hline & & 2.0 & $-1.60159 \times 10^{1}$ & $-1.60159 \times 10^{1}$ & $<1 \times 10^{-4}$ & 3.996 & 4.000 & -0.969 \\
\hline & & 5.0 & $-1.00003 \times 10^{2}$ & $-1.00003 \times 10^{2}$ & $<1 \times 10^{-4}$ & 10.000 & 10.000 & -0.995 \\
\hline
\end{tabular}

are not visible in the plot. Examination of the actual values shows that the variational values lie slightly below the diagonalization results for $g \lesssim 0.61$, and slightly above for larger couplings; at all couplings the results of the three-parameter calculation lie closer to the diagonalization results than the two-parameter variational results. The quality of the results obtained indicates that these variational wavefunctions are very close to the exact ground-state wavefunction for the Rabi Hamiltonian.

As a final remark, it is often useful to express the wavefunctions for Hamiltonians such as the Rabi Hamiltonian in terms of their expansion in a basis of products of oscillator and two-level states, as these are often the form in which initial conditions are formulated. We present here the expansions for the three-parameter ansätze for the ground and first excited states,

$$
\begin{aligned}
& \left|\Psi_{\mathrm{V}}^{(\mathrm{p})}\left(x_{1}, x_{2}, v\right)\right\rangle \\
& \quad=A_{i^{\prime}}\left[A_{1,+} \exp \left[-\frac{1}{2} x_{1}^{2}\right] \sum_{n=0}^{\infty} \frac{x_{1}^{2 n}}{\sqrt{(2 n) !}}|2 n\rangle|\downarrow\rangle\right. \\
& \left.\quad+v A_{2,-} \exp \left[-\frac{1}{2} x_{2}^{2}\right] \sum_{n=0}^{\infty} \frac{x_{2}^{2 n+1}}{\sqrt{(2 n+1) !}}|2 n+1\rangle|\uparrow\rangle\right]
\end{aligned}
$$

and

$$
\begin{aligned}
& \left|\Psi_{\mathrm{V}}^{(\mathrm{n})}\left(x_{1}, x_{2}, v\right)\right\rangle \\
& \quad=A_{l^{\prime}}\left[A_{1,-} \exp \left[-\frac{1}{2} x_{1}^{2}\right] \sum_{n=0}^{\infty} \frac{x_{1}^{2 n+1}}{\sqrt{(2 n+1) !}}|2 n+1\rangle|1\rangle\right. \\
& \left.\quad+v A_{2,+} \exp \left[-\frac{1}{2} x_{2}^{2}\right] \sum_{n=0}^{\infty} \frac{x_{2}^{2 n}}{\sqrt{(2 n) !}}|2 n\rangle|\uparrow\rangle\right]
\end{aligned}
$$

The expansions for the two-parameter states can be found by setting $x_{1}=x_{2}$ in these equations. 


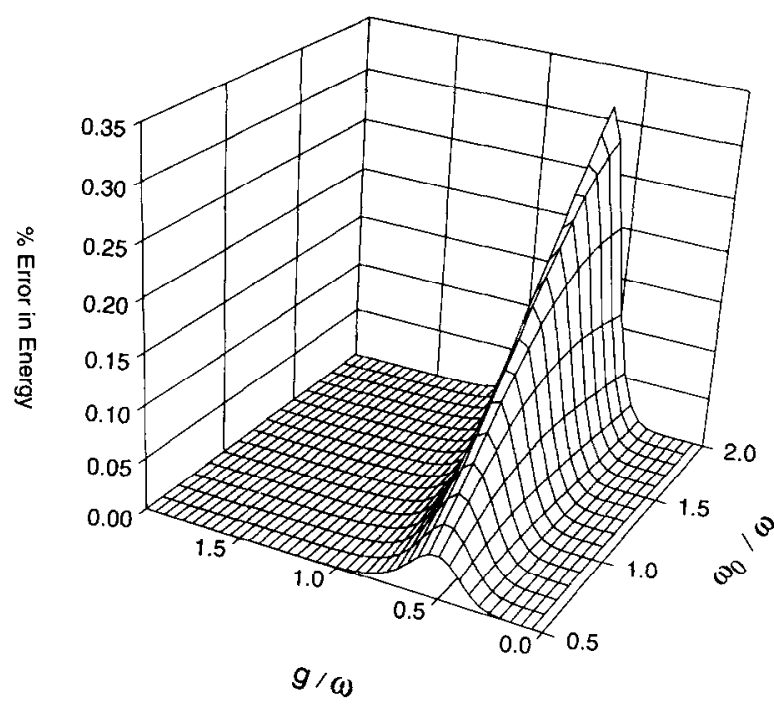

Fig. I. The percentage error in the ground-state energy of the Rabi Hamiltonian obtained from the three-parameter PBV ansatz (11), $\vec{C}_{\mathrm{V}, 0}^{\mathrm{P} 3}$, compared to the results of a large-scale diagonalization as a function of the coupling $g / \omega$ and the two-level splitting $\omega_{0} / \omega$.

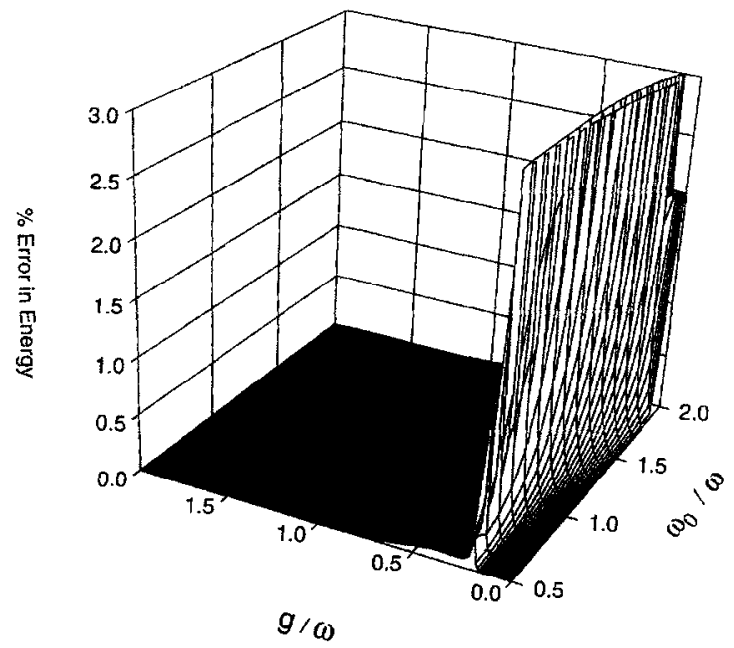

Fig. 2. The percentage error in the first excited state energy of the Rabi Hamiltonian obtained from the three-parameter PBV ansatz (11). $E_{V, 1}^{\mathrm{P3}}$, compared to the results of a large-scale diagonalization as a function of the coupling $g / \omega$ and the two-level splitting $\omega_{0} / \omega$. Note that we have had to truncate the plot in the vertical direction since one obtains an infinite percentage error when the energy becomes zero.

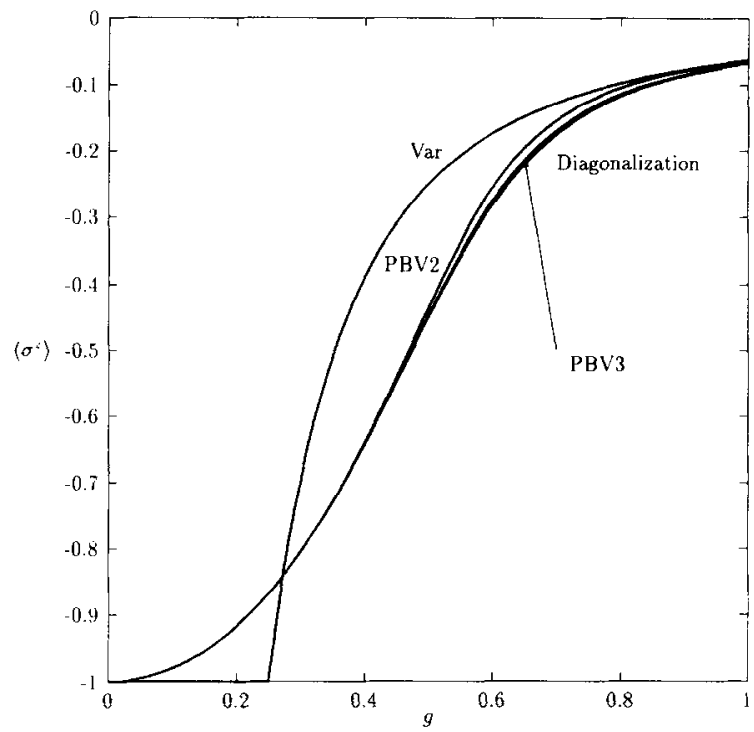

Fig. 3. Expectation values of $\sigma^{z}$ in the two-parameter PBV ground state (9), denoted by PBV2, and the three-parameter PBV ground state (11), denoted by PBV3, compared to its values from a large-scale diagonalization and for the non-projected variational ansatz (4), denoted by Var, for the resonance case $\omega=\omega_{0}=1$.

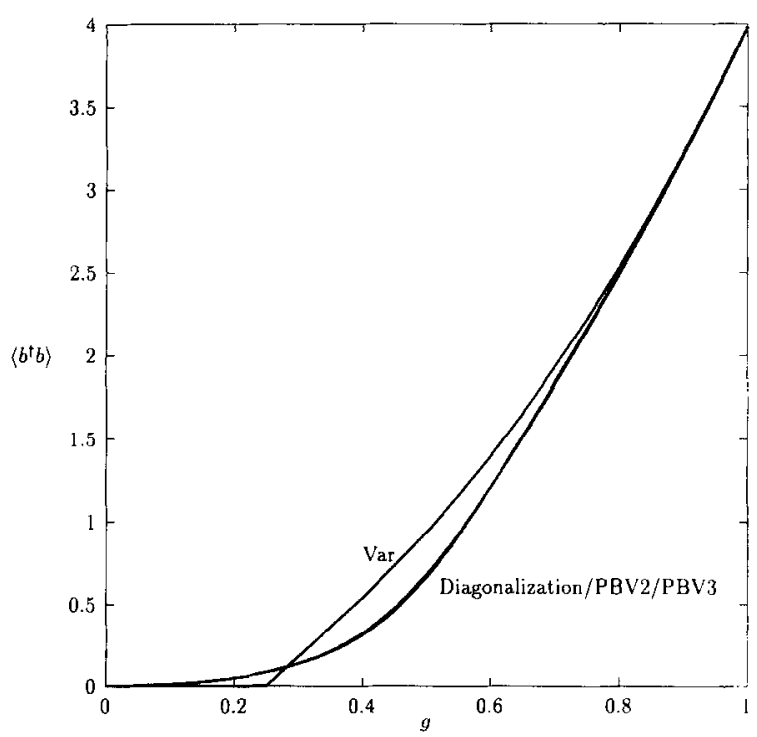

Fig. 4. Expectation values of $b^{\dagger} b$ in the two-parameter PBV ground state (9), denoted by PBV2, and the three-parameter PBV ground state (11), denoted by PBV3, compared to its values from a large-scale diagonalization and for the non-projected variational ansatz (4), denoted by Var, for the resonance case $\omega=\omega_{0}=1$. 


\section{Conclusions}

Our results shown in Tables 1 and 3 and Fig. 1 clearly indicate the improvement that can be achieved in the ground-state cnergy of the Rabi Hamiltonian by performing a projection of the variational ansatz onto a state of positive parity before the variation is carried out. The additional computational effort which the use of the PBV ansätze requires is small considering the dramatic improvement which we obtain in the energy. It is also satisfying to note that our results are very good for a wide range of frequencies and couplings, with maximum error in the three-parameter variation of the order $0.1 \%$, this emphasizes the general utility of the ansätze. The results are particularly good at large couplings and near resonance.

The projected excited state ansätze are seen to be very efficient for a wide range of couplings for cases where $\omega \gtrsim \omega_{0}$. It should be noted that the very large percentage error found for $g=0.2, \omega_{0}=1$ is artificial. It occurs because the excited-state energy for these parameters lies very close to zero; the absolute error is still small.

For $\omega \gtrsim 2 \omega_{0}$, the results for small couplings for the two-parameter PBV ansatz are not very good. This is easy to understand, however, since our ansatz does not include any correlated two-boson excitation (squeezing), which are surely important for the Rabi system near the two-boson resonance $\omega_{0}=2 \omega$. It is possible to include such excitations in the ansatz without too much extra effort, but such inclusions are not necessary for systems near the one-boson resonance $\omega_{0}=$ $\omega$. For cases where $\omega_{0}$ is very much larger than $\omega$, it is necessary to determine which $n$-boson correlations need to be included in the variational ansatz.

For the three-parameter ansatz, the excited state results, shown in Table 4 and Fig. 2, show considerable improvement over those obtained with the twoparameter ansatz, particularly at small couplings. The maximum percentage error for $\omega_{0}=2 \omega$ for the threeparameter variation is of the order of $2 \%$, as opposed to the $40 \%$ found for the two-parameter calculation. Again, near $n$-boson resonances the addition of the relevant $n$-boson correlations to the ansatz should lead to a further reduction in the error, but for many calculations the present ansatz is probably sufficient.

We note that the ground-state energy is accurately given by the two-parameter ansatz and that the three- parameter ansatz does not significantly improve these results. Furthermore an examination of the variational parameters for the two ansätze shows that $x_{1.0 p t} \simeq$ $x_{2 . \mathrm{opt}} \simeq x_{\mathrm{opt}}$. This indicates that the ground state is a singly peaked function in $x$-space. The same is cer tainly not true for the first excited state where $x_{1 . \mathrm{opt}}$ and $x_{2, o p t}$ are significantly different both from each other and from $x_{\mathrm{opt}}$, and the three-parameter ansatr. is essential to obtain good values for the energy. This suggests that the first excited state is doubly peaked in $x$-space. We might therefore conjecture that the wavefunction for the $n$th state requires in total $n+1$ parameters, where the ground state corresponds to $n=1$.

The results which we have obtained for $\left\langle\sigma^{-}\right\rangle$and $\left\langle b^{\dagger} b\right\rangle$, displayed in Figs. 3 and 4 . suggest that the projected ansätze for the ground-state wavefunction, given by Eqs. (9) and (11), are good approximations for the exact ground-state wavefunction. and thus that our ansätze should also be useful for the calculation of expectation values for operators other than the Hamiltonian.

To conclude, we have presented simple two- and three-parameter variational ansätze for the groundstate wavefunction of the Rabi Hamiltonian in which the parity symmetry of the wavefunction is explicitly included in the ansatz. The energies obtained from the three-parameter calculation in particular are very close to the results of a large-scale diagonalization of the Hamiltonian, and are considerably better than those obtained from a non-projected ansat7. We have also presented results for the energy of the first excited state found from a similar ansatz for the opposite parity sector of the Rabi Hamiltonian; the results for this energy are very good near resonance. The necessary modifications to the ansatz required to handle systems far from resonance are also obvious. Finally, our results for $\left\langle\sigma^{i}\right\rangle$ and $\left\langle b^{\dagger} b\right\rangle$ suggest that our three-parameter PBV ansatz for the ground-state wavefunction is probably a very good approximation to the exact ground state. The application of this simple variational ansatz. in time- and temperature-dependent situations should be of considerable future interest.

\section{Acknowledgement}

RFB acknowledges the support of the EPSRC, UK. NJD, RMQ and DvdW acknowledge the support of 
the FRD, South Africa. We thank the referee for useful comments.

\section{References}

$11 \mid$ E.T. Jaynes, F.W. Cummings, Proc. IEEE 51 (1963) 89.

12) P.W. Milonni, S. Singh, Adv. At. Mol. Opt. Phys. 28 (1991) 75 ;

B.W. Shore, P.L. Knight, J. Mod. Opt. 40 (1993) 1195.
[3] P.W. Milonni, J.R. Ackerhalt, H.W. Galbraith, Phys. Rev. Lett. 50 (1983) 966 :

M. Kuś, Phys. Rev. Lett. 54 (1985) 1343.

[4] B.R. Judd, J. Chem. Phys. 67 (1977) 1174

[5] H.G. Reik, P. Lais, M.E. Stützle, M. Doucha, J. Phys. A 20 (1987) 6327

[6] G. Qin, K.-L. Wang, T.-Z. Li, R.-S. Han, M. Feng. Phys. Lett A 239 (1998) 272 .

[7] R.F. Bishop, N.J. Davidson, R.M. Quick, D.M. van der Walt. Phys. Rev. A 54 (1996) R4657. 